\title{
Chain Geometry Determined by the Affine Group
}

\author{
Krzysztof Prażmowski and Aneta Sulima
}

\begin{abstract}
Chain geometry associated with an affine group and with a linear group is studied. In particular, closely related to the respective chain geometries affine partial linear spaces and generalizations of sliced spaces are defined. The automorphisms of thus obtained structures are determined.
\end{abstract}

Mathematics Subject Classification (2010). Primary 51B99, 51A45; Secondary 51B20.

Keywords. Chain geometry, affine transformation, linear group, affine partial linear space, sliced space.

\section{Introduction}

The most general attempt to chain geometry associated with a family of transformations was presented in [7] (see also [4]). Following this approach we consider incidence structures whose blocks are the graphs of a family of bijections. Restricting the class of admissible families of bijections we arrive to some more regular and rich geometries. Particularly, 2-rigid and 3-rigid families are commonly considered (cf. e.g. $[1,6,10])$ and the corresponding incidence geometries are well known.

Still, there are quite well known transformation groups which are neither 2- nor 3-rigid and whose graphs yield interesting incidence structures. In this note we discuss geometry associated with the group of linear bijections of a vector space and the group of affine transformations of an affine space. It turns out that in terms of the corresponding incidence structures with graphs as blocks one can define other incidence structures with lines, which can be obtained from affine and projective spaces by omitting some points and lines. 
Such an incidence structure is called a reduct (of the underlying affine/projective space).

The first arising class consists of line-reducts of affine spaces obtained by deleting the lines parallel to one of a pair of fixed complementary affine subspaces. Formally, one can consider the construction of such reducts as a generalization of the construction presented in [2]; at any rate corresponding reducts are partial affine spaces (cf. [3,9]). The second class consists of structures obtained by deleting from a projective space the points on a pair of complementary subspaces and the lines which cross any of these subspaces. Again, this construction generalizes the known construction of a sliced space (cf. $[5,8])$. The underlying affine and projective space can be recovered from the corresponding reduct. From this we easily characterize the automorphisms of the structure of graphs of the linear and of the affine bijections.

\section{Definitions}

Let us start with the notation and notions used in the paper. Let $\mathbb{W}$ be a vector space; we write

- $G L(\mathbb{W})$ for the group of linear bijections of $\mathbb{W}$,

- $\Gamma L(\mathbb{W})$ for the group of semilinear bijections of $\mathbb{W}$,

- $\operatorname{Tr}(\mathbb{W})$ for the group of all the translations defined in $\mathbb{W}$ i.e. of the maps $\tau_{u}: v \mapsto v+u$ for all vectors $u, v$ of $\mathbb{W}$,

- $G A(\mathbb{W})=\operatorname{Tr}(\mathbb{W}) \circ G L(\mathbb{W})$ for the group of affine maps of the affine space $A G(\mathbb{W})$ over $\mathbb{W}$,

- $\Gamma A(\mathbb{W})=\operatorname{Tr}(\mathbb{W}) \circ \Gamma L(\mathbb{W})$ for the group $\operatorname{Aut}(A G(\mathbb{W}))$ of collineations of $A G(\mathbb{W})$,

- $G P(\mathbb{W})$ for the group of projective collineations of the projective space $P G(\mathbb{W})$; it coincides with the group $G L(\mathbb{W})$ acting on the 1-dimensional subspaces of $\mathbb{W}$,

- $\quad \Gamma P(\mathbb{W})$ for the group of all the collineations of $P G(\mathbb{W})$; it coincides with the action of $\Gamma L(\mathbb{W})$ on 1 -subspaces of $\mathbb{W}$.

Each $f \in \Gamma A(\mathbb{W})$ is associated with a unique automorphism $f^{b}$ of the underlying division ring such that $f=\tau \varphi, \tau$ is a translation, and $\varphi \in \Gamma L(\mathbb{W})$ is $f^{b}$-semilinear.

Let $\mathbb{V}=(V, \theta,+, \cdot)$ be a fixed vector space over a division ring $\mathfrak{F}$ and $\mathbb{Y}=\mathbb{V} \times \mathbb{V}$. In a standard way with a family of transformations $\mathcal{F} \subset V^{V} \subset$ $\wp(V \times V)$ we associate the structure $\mathbf{M}(\mathcal{F})$ of the graphs of $\mathcal{F}$ :

$$
\mathbf{M}(\mathcal{F})=\left(V \times V, \mathcal{F}, \mathcal{L}^{+}, \mathcal{L}^{-}\right),
$$

where $\mathcal{L}^{+}=\{\{u\} \times V: u \in V\}$ and $\mathcal{L}^{-}=\{V \times\{u\}: u \in V\}$. Recall that a function $f \in \mathcal{F}$ is identified with its graph $f=\{(u, f(u)): u \in V\}$, so the two conditions ' $(u, v) \in f^{\prime}$ ' and ' $f(u)=v$ ' are equivalent for $u, v \in V$. 
In accordance with general theory (cf. e.g. [1, Chap. III, §4]) the following holds

Fact 2.1. Let $a \in V \times V$ be a point of $\mathbf{M}(\mathcal{F}), f \in \mathcal{F}, L_{1} \in \mathcal{L}^{+}, L_{2} \in \mathcal{L}^{-}$.

(i) There are $M_{1} \in \mathcal{L}^{+}, M_{2} \in \mathcal{L}^{-}$such that $a \in M_{1}, M_{2}$;

(ii) $\left|L_{1} \cap L_{2}\right|=1$;

(iii) $\left|f \cap L_{1}\right|=1=\left|f \cap L_{2}\right|$.

In the context of general chain geometries we write $a^{\prime} \|^{+} a^{\prime \prime}$ when $a^{\prime}, a^{\prime \prime}$ are on a block in $\mathcal{L}^{+}$, and $a^{\prime} \|^{-} a^{\prime \prime}$ when they are on a block in $\mathcal{L}^{-}$.

For points $a, b$ of $\mathbf{M}(\mathcal{F})$ we say that they are joinable and we write $a \sim b$ when there is $f \in \mathcal{F}$ with $a, b \in f$.

In what follows we shall be mainly concerned with the structure $\mathbf{M}(\mathcal{F})$, where $\mathcal{F}=G A((\mathbb{V}))$ and $\mathcal{F}=G L((\mathbb{V}))$. Actually, we shall investigate the structure

$$
\mathbf{M}^{*}(\mathcal{F})=(V \times V, \mathcal{F}),
$$

which is a bit weaker (formally) than $\mathbf{M}(\mathcal{F})$.

\section{Chains Determined by the Affine Group}

Let $n=\operatorname{dim}(\mathbb{V}) \geq 1$. Note that $\mathbb{Y}$ carries the structure of a $2 n$-dimensional vector space, $\mathcal{L}^{+}$and $\mathcal{L}^{-}$are two directions of affine $n$-subspaces of $A G(\mathbb{Y})$, and the graphs in $G A(\mathbb{V})$ are affine $n$-subspaces of $A G(\mathbb{Y})$ as well. Set $V^{+}=V \times\{\theta\}$ and $V^{-}=\{\theta\} \times V$ for the direction subspaces of $\mathcal{L}^{+}$and $\mathcal{L}^{-}$respectively. Clearly, $\mathbb{Y}$ is the direct sum $\mathbb{Y}=V^{+} \oplus V^{-}$. For distinct affine points $a, b$ we write $\overline{a, b}$ for the affine line which joins $a$ and $b$.

Let $\mathcal{F}=G A(\mathbb{V})$; with $a=\left(u_{1}, u_{2}\right), b=\left(v_{1}, v_{2}\right)$ and $u_{i}, v_{i} \in V$ we have $a \sim b$ iff there is an affine map $f$ such that $f\left(u_{1}\right)=u_{2}$ and $f\left(v_{1}\right)=v_{2}$. Since the group $G A(\mathbb{V})$ acts 2-transitively on $V$ we get

Lemma 3.1. Let $a=\left(u_{1}, u_{2}\right), b=\left(v_{1}, v_{2}\right)$ and $u_{i}, v_{i} \in V$. Clearly, $a=b$ yields $a \sim b$.

Assume that $a \neq b$. Then $a \sim b$ iff $u_{1} \neq v_{1}$ and $u_{2} \neq v_{2}$.

In view of Lemma 3.1, the following conditions are, clearly, equivalent:

$-a \not b$

$-\quad u_{1}=v_{1}$ or $u_{2}=v_{2}$

- $\overline{a, b} \| V^{+}$or $\overline{a, b} \| V^{-}$;

- $a \|^{+} b$ or $a \|^{-} b$.

This gives immediately that for $a \neq b, a \not b$ we have

$$
\{c: c \not a, b\}=\left\{\begin{array}{ll}
a+V^{+} \in \mathcal{L}^{+} & \text {when } a \|^{+} b \\
a+V^{-} \in \mathcal{L}^{-} & \text {when } a \|^{-} b
\end{array} .\right.
$$

In particular, we have

Corollary 3.2. The set $\left\{\mathcal{L}^{-}, \mathcal{L}^{+}\right\}$can be defined in $\mathbf{M}^{*}(G A(\mathbb{V}))$. 
Lemma 3.3. Let $a \neq b, a \sim b$. Then

$$
\left(\bigcap_{f \in G A(\mathbb{V}): a, b \in f} f\right)=\overline{a, b} .
$$

Proof. Write $a=\left(u^{\prime}, u^{\prime \prime}\right), b=\left(v^{\prime}, v^{\prime \prime}\right)$, and $\Phi_{a, b}=\{f \in G A(\mathbb{V}): a, b \in f\}=$ $\left\{f \in G A(\mathbb{V}): f\left(u^{\prime}\right)=u^{\prime \prime}, f\left(v^{\prime}\right)=v^{\prime \prime}\right\}$. By the assumptions, $u^{\prime} \neq v^{\prime}$ and $u^{\prime \prime} \neq v^{\prime \prime}$. Then

$$
\begin{aligned}
& \left(\bigcap_{f: f \in \Phi_{a, b}} f\right)= \\
& \quad\left\{(x, y): \forall f \in G A(\mathbb{V})\left[f\left(u^{\prime}\right)=u^{\prime \prime} \wedge f\left(v^{\prime}\right)=v^{\prime \prime} \Longrightarrow f(x)=y\right]\right\}=: \mathcal{X} .
\end{aligned}
$$

Clearly, each $f \in \mathcal{Z}_{a, b}$ maps the line $\overline{u^{\prime}, v^{\prime}}$ onto $\overline{u^{\prime \prime}, v^{\prime \prime}}$. Let $x \notin \overline{u^{\prime}, v^{\prime}}, y \notin \overline{u^{\prime \prime}, v^{\prime \prime}}$. Then there is $f \in G A(\mathbb{V})$ such that $f\left(u^{\prime}\right)=u^{\prime \prime}, f\left(v^{\prime}\right)=v^{\prime \prime}$, and $f(x) \neq y$, and thus $(x, y) \notin \mathcal{X}$. So, $\mathcal{X} \subset \overline{u^{\prime}, v^{\prime}} \times \overline{u^{\prime \prime}, v^{\prime \prime}}$.

Since each affine map preserves the ratio, $f\left(u^{\prime}\right)=u^{\prime \prime}, f\left(v^{\prime}\right)=v^{\prime \prime}$ yields $f\left(\lambda u^{\prime}+(1-\lambda) v^{\prime}\right)=\lambda u^{\prime \prime}+(1-\lambda) v^{\prime \prime}$ for each scalar $\lambda$. Note that $\overline{u^{\prime}, v^{\prime}}=\left\{\lambda u^{\prime}+(1-\lambda) v^{\prime}: \lambda\right.$ a scalar $\}$. Thus $\mathcal{X}=\left\{\left(\lambda u^{\prime}+(1-\lambda) v^{\prime}, \lambda u^{\prime \prime}+(1-\right.\right.$ $\left.\lambda) v^{\prime \prime}\right): \lambda$ a scalar $\}=\{\lambda a+(1-\lambda) b: \lambda$ a scalar $\}=\overline{a, b}$ as required.

\subsection{Two-Directions Reduct of an Affine Space}

Let a vector space $\mathbb{Y}$ be the direct sum of its two subspaces $V^{+}$and $V^{-}$, where $\operatorname{dim}\left(V^{+}\right), \operatorname{dim}\left(V^{-}\right) \geq 1$. Then $V^{+}+V^{-}$is the set of vectors of $\mathbb{Y}$. Let us emphasize that we do not assume $\operatorname{dim}\left(V^{+}\right)=\operatorname{dim}\left(V^{-}\right)$.

Without loss of generality we can write $\mathbb{Y}$ in the form $\mathbb{Y}=V_{0}^{+} \times V_{0}^{-}$for vector spaces $V_{0}^{+}, V_{0}^{-}, V^{+}=V_{0}^{+} \times\left\{\theta_{2}\right\}$, and $V^{-}=\left\{\theta_{1}\right\} \times V_{0}^{-}$. In mathematical practice, these two approaches are frequently mixed and the spaces in pairs $V_{0}^{+}, V^{+}$and $V_{0}^{-}, V^{-}$are identified. Set $\mathcal{L}^{+}:=V^{-}+\left\{V^{+}\right\}=\left\{u+V^{+}: u \in V^{-}\right\}$ and $\mathcal{L}^{-}=V^{+}+\left\{V^{-}\right\}$. Write $\mathcal{L}_{0}$ for the class of lines of the affine space

$$
A G(\mathbb{Y})=: \mathfrak{A}
$$

that are not parallel neither to $V^{+}$nor to $V^{-}$and set

$$
\mathbf{B}\left(V^{+}, V^{-}\right):=\left(V^{+}+V^{-}, \mathcal{L}_{0}\right) .
$$

With fixed $V^{+}, V^{-}$we simply write $\mathfrak{B}$ instead of $\mathbf{B}\left(V^{+}, V^{-}\right)$. The structure $\mathbf{B}\left(V^{+}, V^{-}\right)$will be referred to as a two-directions reduct of $\mathfrak{A}$.

Then Corollary 3.2, (2), and Lemma 3.3 state the following

Corollary 3.4. Assume that $V_{0}^{+}=V=V_{0}^{-}$. The family $\mathcal{L}_{0}$ and the pair $\left\{\mathcal{L}^{+}, \mathcal{L}^{-}\right\}$are definable in $\mathbf{M}^{*}(G A(\mathbb{V}))$. In particular, the structure $\mathbf{B}(V \times$ $\{\theta\},\{\theta\} \times V)$ is definable in $\mathbf{M}^{*}(G A(\mathbb{V}))$.

Clearly, $\mathbf{B}\left(V^{+}, V^{-}\right)$is a partial linear space. For points $a, b$ of an arbitrary partial linear space $\mathfrak{D}$ we write $a \sim b$ when they are collinear i.e. when they are on a line of $\mathfrak{D}$. In this subsection we shall apply this definition to 
the points of $\mathbf{B}\left(V^{+}, V^{-}\right)=: \mathfrak{B}$. In the particular case when $\mathfrak{B}$ is determined by the structure $\mathbf{M}^{*}(G A(\mathbb{V}))$ as in Corollary 3.4 , the binary collinearity of $\mathfrak{B}$ and the relation of joinability in $\mathbf{M}^{*}(G A(\mathbb{V}))$ introduced in Sect. 2 coincide (cf. Lemma 3.1), so we can use the same symbol to denote them. What is more, Lemma 3.1, Corollary 3.2, and formula (1) remain valid in an arbitrary two-directions reduct $\mathbf{B}\left(V^{+}, V^{-}\right)$.

Let $\mathcal{P}$ be the class of affine planes in $\mathfrak{A}$. The following is just an easy observation.

Lemma 3.5. Let $\pi \in \mathcal{P}$ and let $\mathcal{B}$ be the set of affine lines that lie on $\pi$ and do not belong to $\mathcal{L}_{0}$. Then one of the following holds:

(i) $\mathcal{B}=\emptyset$,

(ii) $\mathcal{B}$ is a parallel pencil on $\pi$,

(iii) $\mathcal{B}$ is the union of two parallel pencils on $\pi$,

(iv) all the lines on $\pi$ are in $\mathcal{B}$.

In each case except (iv), in which $\pi \| V^{+}$or $\pi \| V^{-}, \pi$ contains a triangle with the sides in $\mathcal{L}_{0}$.

Assume that $\operatorname{dim}(\mathbb{Y}) \geq 3$. Then for every affine line $L \notin \mathcal{L}_{0}$ there are planes $\pi_{1}, \pi_{2}$ such that $L=\pi_{1} \cap \pi_{2}$ and $\pi_{i}$ satisfies either (i) or (ii) for both $i=1$ and $i=2$.

Lemma 3.6. Let $\pi \in \mathcal{P}$ and let $L_{1}, L_{2}, L_{3} \in \mathcal{L}_{0}$ yield a triangle in $\pi$. Assume that the coordinate division ring of $\mathbb{Y}$ contains at least 5 elements. Then

$$
\left(\bigcup_{L \in \mathcal{L}_{0}:\left|L \cap\left(L_{1} \cup L_{2} \cup L_{3}\right)\right| \geq 2} L\right)=\pi .
$$

Proof. Set $\mathcal{X}=\left(\bigcup_{L \in \mathcal{L}_{0}:\left|L \cap\left(L_{1} \cup L_{2} \cup L_{3}\right)\right| \geq 2} L\right)$; clearly, $\mathcal{X} \subset \pi$. Let $a_{1}, a_{2}, a_{3}$ be the vertices of our triangle, $a_{i} \notin L_{i}$ for $i=1,2,3$. Let $x \in \pi$. By Lemma 3.5, there are at most two affine lines through $x$ not in $\mathcal{L}_{0}$ and thus there is at least one line $M$ in $\mathcal{L}_{0}$ that is contained in $\pi$, goes through $x$, and crosses $L_{1}$ in a point $c$ distinct from $a_{2}, a_{3}$. Clearly, $c \notin L_{2}, L_{3}$. Then $M \nVdash L_{2}$ or $M \nVdash L_{3}$, so $M$ crosses a second side of our triangle in a point distinct from $c$ and thus $x \in \mathcal{X}$.

Since Lemma 3.6 plays an important role in further investigations, from now on till the end of Sect. 3 we assume that

the coordinate division ring of $\mathbb{Y}$ is not $G F(2), G F(3)$, and $G F(4)$.

Write $\mathcal{L}$ for the class of all the lines of $\mathfrak{A}$. From Lemma 3.6 we deduce immediately that the class $\mathcal{P}_{0}:=\left\{\pi \in \mathcal{P}: \pi \nVdash V^{+}, V^{-}\right\}$is definable in $\mathfrak{B}$ and from Lemma 3.5 we get that the set $\left\{\pi_{1} \cap \pi_{2}: \pi_{1}, \pi_{2} \in \mathcal{P}_{0}, \pi_{1} \neq \pi_{2},\left|\pi_{1} \cap \pi_{2}\right| \geq 2\right\}=\mathcal{L}$ is definable as well. This, finally, gives

Proposition 3.7. Let $\operatorname{dim}\left(V^{+} \oplus V^{-}\right) \geq 3$. The affine geometry $A G\left(V^{+} \oplus V^{-}\right)$ can be defined in the two-directions reduct $\mathbf{B}\left(V^{+}, V^{-}\right)$. 


\subsection{Automorphisms}

Clearly, $\operatorname{Tr}(\mathbb{Y}) \subset \operatorname{Aut}\left(\mathbf{B}\left(V^{+}, V^{-}\right)\right)$. Moreover, if $\operatorname{dim}\left(V^{+}\right)=\operatorname{dim}\left(V^{-}\right)$then without loss of generality we can assume $V^{+} \cong V \cong V^{-}, \mathbb{Y}=V \times V$, and then the map $\sigma:(u, v) \mapsto(v, u), u, v \in V$ is a linear bijection which preserves the set $\left\{V^{+}, V^{-}\right\}$and therefore it is an automorphism of $\mathbf{B}\left(V^{+}, V^{-}\right)$.

Remark. It is seen that $\sigma \in \operatorname{Aut}\left(\mathbf{M}^{*}(G A \mathbb{V})\right)$ as well. Indeed, if $f \in G A(\mathbb{V})$ then $\sigma(f)$ is the graph of $f^{-1}$.

In view of Proposition 3.7, a characterization of the whole group $\operatorname{Aut}\left(\mathbf{B}\left(V^{+}, V^{-}\right)\right)$is easy.

Proposition 3.8. Let $\operatorname{dim}(\mathbb{Y}) \geq 3$ and $F$ be a map. Then $F \in \operatorname{Aut}\left(\mathbf{B}\left(V^{+}, V^{-}\right)\right.$ iff $F \in \Gamma A(\mathbb{Y})$ and $F$ preserves the set $\left\{\mathcal{L}^{+}, \mathcal{L}^{-}\right\}$.

If $\operatorname{dim}\left(V^{+}\right) \neq \operatorname{dim}\left(V^{-}\right)$the last condition is equivalent to the following: ' $F$ preserves two directions $\mathcal{L}^{+}$and $\mathcal{L}^{-}$of subspaces of $\mathfrak{A}$ '.

In case $\operatorname{dim}\left(V^{+}\right)=\operatorname{dim}\left(V^{-}\right)$i.e. when $\mathbf{B}\left(V^{+}, V^{-}\right)$is derived from $\mathbf{M}^{*}(G A(\mathbb{V}))$ (cf. Corollary 3.4$)$, Proposition 3.8 yields an immediate analytical characterization of the automorphisms of the chains of the affine group.

Lemma 3.9. The following conditions are equivalent:

(i) $F \in \operatorname{Aut}\left(\mathbf{M}^{*}(G A(\mathbb{V}))\right)$

(ii) There are $u_{1}, u_{2} \in V$ and $\phi_{1}, \phi_{2} \in \Gamma L(\mathbb{V})$ such that $\phi_{2} \phi_{1}^{-1} \in G L(\mathbb{V})$ and $F$ is determined by one of the following formulas

$$
\begin{aligned}
& F\left(x_{1}, x_{2}\right)=\left(\phi_{1}\left(x_{1}\right)+u_{1}, \phi_{2}\left(x_{2}\right)+u_{2}\right) \\
& F\left(x_{1}, x_{2}\right)=\left(\phi_{2}\left(x_{2}\right)+u_{2}, \phi_{1}\left(x_{1}\right)+u_{1}\right)
\end{aligned}
$$

for all $x_{1}, x_{2} \in V$.

Remark. Let a map $F$ in $\Gamma A(\mathbb{Y})$ be defined by (4). Equivalently, we can write

$$
\left.F\left(x_{1}, x_{2}\right)=\left(f_{1}\left(x_{1}\right), f_{2}\left(x_{2}\right)\right), \quad \text { (we write also } F=f_{1} \times f_{2}\right)
$$

where $f_{1}, f_{2} \in \Gamma A(\mathbb{V})$ and $f_{1}^{b}=F^{b}=f_{2}^{b}$. Note that $f_{2} f_{1}^{-1} \in G A(\mathbb{V})$ iff $F$ preserves $G A(\mathbb{V})$.

Lemma 3.10. Let $f_{1}, f_{2} \in \Gamma A(\mathbb{V})$. The following conditions are equivalent:

(i) $f_{2} f_{1}^{-1} \in G A(\mathbb{V})$;

(ii) $f_{1} f_{2}^{-1} \in G A(\mathbb{V})$;

(iii) $f_{2}^{-1} f_{1} \in G A(\mathbb{V})$.

Proof. Clearly, $f_{1} f_{2}^{-1}=\left(f_{2} f_{1}^{-1}\right)^{-1}$, which justifies (i) $\Longleftrightarrow$ (ii). The relation $\left(f_{2}^{-1} f_{1}\right)^{f_{2}}=f_{2} f_{2}^{-1} f_{1} f_{2}^{-1}=f_{1} f_{2}^{-1}$ justifies (ii) $\Longleftrightarrow($ iii).

Let $F \in \Gamma A(\mathbb{Y})$ be defined by (6). By Lemma 3.10, this formula can be rewritten in the form

$$
F\left(x_{1}, x_{2}\right)=\left(f\left(x_{1}\right), g f\left(x_{2}\right)\right) \text { with } f \in \Gamma A(\mathbb{V}), g \in G A(\mathbb{V}) .
$$

and therefore $F$ can be identified with the pair $(f, g) \in \Gamma A(\mathbb{V}) \times G A(\mathbb{V})$ such that $F=f \times g f$. Computing the composition rule 


$$
\left(f_{2} \times g_{2} f_{2}\right) \circ\left(f_{1} \times g_{1} f_{1}\right)=f_{2} f_{1} \times\left(g_{2} g_{1} f_{2}\right)\left(f_{2} f_{1}\right)
$$

for $f_{1}, f_{2} \in \Gamma A(\mathbb{V}), g_{1}, g_{2} \in G A(\mathbb{V})$ we, finally, prove the following

Proposition 3.11. The class of maps defined by (6), which coincides with the group $\operatorname{Aut}(\mathbf{M}(G A(\mathbb{V})))$, is isomorphic to $\Gamma A(\mathbb{V}) \ltimes G A(\mathbb{V})$.

Finally, note that an automorphism $F$ defined by (5) can be written in the form $F=\sigma \circ(f \times g f)$ with $f \in \Gamma A(\mathbb{V}), g \in G A(\mathbb{V})$. To complete our computations we note that

$$
\sigma \circ(f \times g f) \circ \sigma=g f \times g^{-1} g f .
$$

\section{Chains Determined by the Linear Group}

Analogous investigations carried out over the groups $G L(\mathbb{V})$ and $\Gamma L(\mathbb{V})$ are more complex. The group $G L(\mathbb{V})$ is the stabilizer of the point $(\theta, \theta)$ in the group $G A(\mathbb{V})$ so, the chains of $\mathbf{M}^{*}(G L(\mathbb{V}))$ are the chains of $\mathbf{M}^{*}(G A(\mathbb{V}))$ which pass through $(\theta, \theta)$. Consequently, the incidence structure $\mathbf{M}^{*}(G L(\mathbb{V}))$ contains an isolated point i.e. such a point that no block of $\mathbf{M}^{*}(G L(\mathbb{V}))$ passes through it. From Lemma 3.1, a point $(u, v) \in \mathbb{Y}$ is not isolated in $\mathbf{M}^{*}(G L(\mathbb{V}))$ iff $(u, v) \notin V^{+} \cup V^{-}$. As an immediate consequence of Lemma 3.3 we obtain the following

Fact 4.1. Let $a$ be a vector of $\mathbb{Y}$ such that $a \notin V^{+} \cup V^{-}$. Then

$$
\left(\bigcap_{f \in G L(\mathbb{V}): a \in f} f\right)=\langle a\rangle,
$$

i.e. it is the one-dimensional subspace of $\mathbb{Y}$ spanned by a. Each one dimensional subspace of $\mathbb{Y}$ not contained in $V^{+} \cup V^{-}$can be presented in this way.

Let $a=\left(u_{1}, u_{2}\right), b=\left(v_{1}, v_{2}\right)$ with $\theta \neq u_{1}, u_{2}, v_{1}, v_{2} \in \mathbb{V}$. Then $a, b \notin$ $V^{+} \cup V^{-}$and thus $a, b$ are not isolated.

Lemma 4.2. Let $a, b$ be as above, assume that $a \neq b$. The following conditions are equivalent

(i) $a \sim b$ (i.e. $a, b$ are joinable in $\mathbf{M}^{*}(G L(\mathbb{Y}))$ )

(ii) either

(a) both $u_{1}, v_{1}$ and $u_{2}, v_{2}$ are linearly dependent in pairs, and then there is $\lambda$ with $\lambda u_{1}=v_{1}$ and $\lambda u_{2}=v_{2}$ or

(b) neither $u_{1}, v_{1}$ nor $u_{2}, v_{2}$ are linearly dependent pairs.

Let $a, b$ be as above. Assume that $\langle a\rangle \neq\langle b\rangle$ and let $U=\langle a, b\rangle$ be the two-dimensional subspace spanned by $a, b$.

Lemma 4.3. The following conditions are equivalent.

(i) $a \not b$;

(ii) the plane $U$ crosses $V^{+}$or $V^{-}$in a non-zero vector. 
Proof. Assume that $a \not b$. In view of Lemma 4.2 this means that there is a scalar $\lambda$ such that $\lambda u_{1}=v_{1}$ and $\lambda u_{2} \neq v_{2}\left(\right.$ or $\lambda u_{1} \neq v_{1}$ and $\left.\lambda u_{2}=v_{2}\right)$. Consider the first case. Let $\beta \neq 0$ be arbitrary, $\alpha=-\beta \lambda$, and $c=\alpha a+\beta b$. Then $U \ni c=\left(\theta, \beta\left(v_{2}-\lambda u_{2}\right)\right) \in V^{+}$and $c \neq(\theta, \theta)$. In the second case, analogously, we find a non zero $c \in U \cap V^{-}$. Thus (i) implies (ii).

Now, let $c \in U \cap V^{+}$be non zero i.e. $c=(\theta, v)=\alpha a+\beta b$ for some $v \neq \theta$ and scalars $\alpha, \beta$. In particular, $\theta=\alpha u_{1}+\beta v_{1}$ and $v=\alpha u_{2}+\beta v_{2}$. Either $\alpha \neq 0$ or $\beta \neq 0$, as $v \neq \theta$. Assume that $\beta \neq 0$, then $v_{1}=\lambda u_{1}$ for a scalar $\lambda$. If there were $v_{2}=\lambda u_{2}$ we get $a=\lambda b$ and either $\lambda=0$ (and, consequently, $a=\theta)$ or $\langle a\rangle=\langle b\rangle$, which both contradict the assumptions. So, $v_{2} \neq \lambda u_{2}$ and, by Lemma 4.2, $a \not b$. In the case $\alpha \neq 0$, analogously, we end up with $a \nsim b$. Finally, analogous reasoning proves (i) when there is a non-zero vector $c \in U \cap V^{-}$.

Finally, as in Lemma 3.3 one can compute

Lemma 4.4. Let $a, b \notin V^{+} \cup V^{-},\langle a\rangle \neq\langle b\rangle$, and $a \sim b$. Then

$$
\left(\bigcap_{f \in G L(\mathbb{V}): a, b \in f} f\right)=\langle a, b\rangle .
$$

Each two-dimensional subspace of $\mathbb{Y}$ which crosses $V^{+} \cap V^{-}$in $(\theta, \theta)$ only can be presented in this form.

\subsection{Two-Holes Sliced Space}

As in Sect. 3.1 we fix a vector space $\mathbb{Y}$ being a direct sum of two its subspaces $V^{+}$and $V^{-}$. And, as in Sect. 3.1 we do not assume that $\operatorname{dim}\left(V^{+}\right)=\operatorname{dim}\left(V^{-}\right)$. Let $\theta$ be the zero vector of $\mathbb{Y}$. Consider the projective space $\mathfrak{P}=P G(\mathbb{Y})$ and its two subspaces

$$
\mathcal{H}^{+}=\left\{\langle u\rangle: \theta \neq u \in V^{+}\right\} \text {and } \mathcal{H}^{-}=\left\{\langle u\rangle: \theta \neq u \in V^{-}\right\} .
$$

Then $\mathcal{H}^{+}$and $\mathcal{H}^{-}$are complementary subspaces: they are disjoint and span the space. Let $\mathcal{S}$ consist of the points of $\mathfrak{P}$ outside $\mathcal{H}^{+} \cup \mathcal{H}^{-}$and let $\mathcal{T}$ consist of the lines of $\mathfrak{P}$ which miss $\mathcal{H}^{+} \cup \mathcal{H}^{-}$. To have $\mathcal{T} \neq \emptyset$ we must assume that neither $\mathcal{H}^{+}$nor $\mathcal{H}^{-}$is a hyperplane, i.e. we assume $\operatorname{dim}\left(V^{+}\right), \operatorname{dim}\left(V^{-}\right) \geq 2$. Finally, we set

$$
\mathbf{T}\left(\mathcal{H}^{+}, \mathcal{H}^{-}\right):=(\mathcal{S}, \mathcal{T})
$$

The structure $\mathbf{T}\left(\mathcal{H}^{+}, \mathcal{H}^{-}\right)$will be called a two-holes sliced space. When $\mathcal{H}^{+}, \mathcal{H}^{-}$are fixed we write simply $\boldsymbol{T}=\mathbf{T}\left(\mathcal{H}^{+}, \mathcal{H}^{-}\right)$. By Fact 4.1 and Lemma 4.4, we obtain immediately the following analogue of Corollary 3.4.

Proposition 4.5. Let $\mathbb{Y}=\mathbb{V} \times \mathbb{V}, V^{+}=V \times\{\theta\}, V^{-}=\{\theta\} \times V$, and $\mathcal{H}^{+}, \mathcal{H}^{-}$ be the corresponding subspaces of $\mathfrak{P}$. The structure $\mathbf{T}\left(\mathcal{H}^{+}, \mathcal{H}^{-}\right)$is definable in $\mathbf{M}^{*}(G L(\mathbb{V}))$. 
The next step is to show that $\mathfrak{P}$ can be reinterpreted in terms of its reduct $\boldsymbol{T}$. This needs some work.

Let us call the projective points in $\mathcal{S}$ proper and those in $\mathcal{H}^{+} \cup \mathcal{H}^{-}$ improper. Let $L$ be a line of $\mathfrak{P}$ not contained in $\mathcal{H}^{+} \cup \mathcal{H}^{-}$. We write $L^{\infty^{+}}$ for the point in $L \cap \mathcal{H}^{+}$and $L^{\infty^{-}}$for $L \cap \mathcal{H}^{-}$, if nonempty; otherwise $L^{\infty^{+}}$ ( $L^{\infty^{-}}$resp.) is $\emptyset$ simply. Let us write $L \in \mathcal{L}_{i}$ when $|L \backslash \mathcal{S}|=i$. Consequently, $L \in \mathcal{L}_{0}$ when $L^{\infty^{+}} \cup L^{\infty^{-}}=\emptyset, L \in \mathcal{L}_{1}$ when either $L^{\infty^{+}}=\emptyset$ and $L^{\infty^{-}}$is a point or $L^{\infty^{-}}=\emptyset$ and $L^{\infty^{+}}$is a point, and $L \in \mathcal{L}_{2}$ when $L^{\infty^{+}}$and $L^{\infty^{-}}$both are points.

Let $A$ be a plane of $\mathfrak{P}$ not contained in $\mathcal{H}^{+} \cup \mathcal{H}^{-}$. Analogously we use the symbols $A^{\infty^{+}}$and $A^{\infty^{-}}$(note that following such an approach we frequently identify a point with the set consisting of this point. We believe that this should not lead to a misunderstanding, though). Note an evident

Fact 4.6. $A^{\infty^{+}}$and $A^{\infty^{-}}$may be empty, a point, or a line. If $\operatorname{dim}\left(V^{+}\right)=2=$ $\operatorname{dim}\left(V^{-}\right)$then $A^{\infty^{+}}$and $A^{\infty^{-}}$is at least a point. It is impossible to have $A^{\infty^{+}}$ and $A^{\infty^{-}}$both a line.

Write $\Pi_{i}$ for the class of planes of $\mathfrak{P}$ on which $i$ points are outside $\mathcal{S}$ with $i=0,1,2$, and let $\Pi=\Pi_{0} \cup \Pi_{1} \cup \Pi_{2}$. Thus $A \in \Pi_{0}$ when $A^{\infty^{+}} \cup A^{\infty^{-}}=\emptyset$, $A \in \Pi_{1}$ when $A^{\infty^{+}}=\emptyset$ and $A^{\infty^{-}}$is a point or $A^{\infty^{-}}=\emptyset$ and $A^{\infty^{+}}$is a point, and $A \in \Pi_{2}$ when $A^{\infty^{+}}$and $A^{\infty^{-}}$both are points. Let us warn that $\Pi$ is not the set of all the planes of $\mathfrak{P}$ not contained in $\mathcal{H}^{+} \cup \mathcal{H}^{-}$.

With elementary geometrical reasoning we obtain two subsequent lemmas.

Lemma 4.7. Let $A$ be a plane of $\mathfrak{P}$. Assume that the size of the lines in $\mathfrak{P}$ is at least 4 .

(i) If $A^{\infty^{+}}$is a line or $A^{\infty^{-}}$is a line then $A$ does not contain any line in $\mathcal{T}$.

(ii) Assume that neither $A^{\infty^{+}}$nor $A^{\infty^{-}}$is a line. Then $A$ contains a triangle of $\mathfrak{T}$ (i.e. with the sides in $\mathcal{T}$ and the vertices in $\mathcal{S}$ ).

(iii) Let $A$ contain a triangle of $\boldsymbol{T}$ with the sides $L_{1}, L_{2}, L_{2}$ in $\mathcal{T}$. Then

$$
\left(\bigcup_{L \in \mathcal{T}:\left|L \cap\left(L_{1} \cup L_{2} \cup L_{3}\right)\right| \geq 2} L\right)=A \backslash\left(A^{\infty^{+}} \cup A^{\infty^{-}}\right) .
$$

In view of Lemma 4.7, as in Sect. 3.1 from now on

till the end of Sect. 4 we adopt the assumption $(\star)$.

Let $\sim$ denote the binary collinearity relation of $\boldsymbol{T}$ and $\nsim$ be its complement.

Lemma 4.8. Let $A$ be a plane of $\mathfrak{P}$. 
(i) Let $A \in \Pi_{1}$, set $Q=A^{\infty^{+}} \cup A^{\infty^{-}}$. Then the noncollinearity relation $\chi$ is transitive on $A \cap \mathcal{S}$ and thus the relation $\chi \cup=i$ an equivalence relation. Its equivalence classes are exactly the sets $L \backslash Q$, where $L$ is a line on $A$ not in $\mathcal{T}$. Each line $L$ of $\mathfrak{P}$ contained in $A$ and not in $\mathcal{T}$ is in $\mathcal{L}_{1}$.

(ii) Let $A, Q$ be as in (i) and $M_{i}=L_{i} \backslash Q$, where $L_{i}$ is a line in $\mathcal{L}_{1}$ contained in $A$ for $i=1,2$. Then $M_{1} \cap M_{2}=\emptyset$ or $M_{1}=M_{2}$.

(iii) Let $A \in \Pi_{2}$. Write $\left\{q^{+}\right\}=A^{\infty^{+}},\left\{q^{-}\right\}=A^{\infty^{-}}$, and $Q=\left\{q^{+}, q^{-}\right\}$. The noncollinearity relation is not transitive on $A \cap \mathcal{S}$. For any two noncollinear points $a, b$ of $\boldsymbol{T}$ which lie on $A$ the set $\{x \in A \cap \mathcal{S}: a, b \neq x\}$ is the set $L \backslash Q$, where $L$ is the projective line which joins the points a, b. Clearly, $L \in \mathcal{L}_{1} \cup \mathcal{L}_{2}$.

(iv) Let $A, Q$ be as in (iii) and $M_{i}=L_{i} \backslash Q$, where $L_{i}$ is a line in $\mathcal{L}_{1} \cup \mathcal{L}_{2}$ contained in $A$ for $i=1,2$. Then the condition " $M_{1} \cap M_{2}=\emptyset$ or $M_{1}=M_{2}$ " holds iff $q^{+} \in L_{1}, L_{2}$ or $q^{-} \in L_{1}, L_{2}$.

From Lemma 4.7, $A \in \Pi$ when $A$ contains a triangle in $\mathfrak{T}$, and the class $\Pi$ is definable in $\boldsymbol{T}$ or, to be more precise, the class

$$
\{A \cap \mathcal{S}: A \in \Pi\}=: \mathcal{P}
$$

is definable in $\boldsymbol{T}$. From Lemma 4.8 (i),(iii), the classes $\mathcal{P}_{i}=\left\{A \cap \mathcal{S}: A \in \Pi_{i}\right\}$ with $i=0,1,2$ are definable as well. In Lemma 4.8 we have obtained

$\left\{L \cap \mathcal{S}: L \in \mathcal{L}_{1} \cup \mathcal{L}_{2}\right\}=\{\{x \in B: x \nsim a, b\}: a \not b, a, b \in B \in \mathcal{P}\}=: \mathcal{M} .(10)$

Note also that the classes $\mathcal{M}_{1}:=\left\{L \cap \mathcal{S}: L \in \mathcal{L}_{1}\right\}$ and $\mathcal{M}_{2}:=\left\{L \cap \mathcal{S}: L \in \mathcal{L}_{2}\right\}$ are distinguishable. Indeed, for $M \in \mathcal{M}$ we have

$$
M \in \mathcal{M}_{1} \text { iff } M \subset B \text { for some } B \in \mathcal{P}_{1} .
$$

To complete our course we need the following (proved with an easy though tedious linear combinatorics)

Lemma 4.9. Let $L_{1}, L_{2} \in \mathcal{L}_{1} \cup \mathcal{L}_{2}$ be distinct. Assume that $L_{1}^{\infty^{+}}=L_{2}^{\infty^{+}}=q$ or $L_{1}^{\infty^{-}}=L_{2}^{\infty^{-}}=q$. Then either the projective plane $A$ through $L_{1}, L_{2}$ is in $\Pi$ or there is a line $L_{3} \in \mathcal{L}_{1}$ through $q, L_{3} \neq L_{1}, L_{2}$ such that both planes: through $L_{3}, L_{1}$ and through $L_{3}, L_{2}$ are in $\Pi$.

The following analogue of a parallelism $M_{1} \| M_{2}$ is definable in terms of geometry of $\boldsymbol{T}$ for $M_{1}, M_{2}$ in the class $\mathcal{M}_{1}$

$$
\begin{aligned}
M_{1} \| M_{2} \Longleftrightarrow M_{1}= & M_{2} \vee(\exists B \in \mathcal{P})\left[M_{1}, M_{2} \subset B \wedge M_{1} \cap M_{2}=\emptyset\right] \\
& \vee\left(\exists M_{3} \in \mathcal{M}_{1}\right)\left(\exists B_{1}, B_{2} \in \mathcal{P}\right)\left[M_{1}, M_{3} \subset B_{1}\right. \\
& \wedge M_{2}, M_{3} \subset B_{2} \\
& \left.\wedge M_{1} \cap M_{3}=\emptyset \wedge M_{2} \cap M_{3}=\emptyset\right] .
\end{aligned}
$$

The relation $\|$ is an equivalence relation.

For $M \in \mathcal{M}$ denote by $\bar{M}$ the line of $\mathfrak{P}$ which contains $M$ and write $M^{\infty}=\bar{M}^{\infty^{+}} \cup \bar{M}^{\infty^{-}}$. The parallel lines in $\mathcal{M}_{1}$ have the same improper point, i.e. $M_{1} \| M_{2}$ iff $M_{1}^{\infty}=M_{2}^{\infty}$. Thus the equivalence classes of the relation $\| \subset \mathcal{M}_{1} \times \mathcal{M}_{2}$ correspond to the points in $\mathcal{H}^{+} \cup \mathcal{H}^{-}$, which were deleted from 
$\mathfrak{P}$ when $\mathfrak{T}$ was defined. Thus, we have re-defined the point set of $\mathfrak{P}$ in terms of $\boldsymbol{T}$. We need to re-define the omitted lines of $\mathfrak{P}$ : those, which are not entirely contained in $\mathcal{H}^{+} \cup \mathcal{H}^{-}$correspond to the elements of $\mathcal{M} \cup \mathcal{T}$. So we need to re-define the incidence of the improper points and the elements of $\mathcal{M}$. By the above, it is immediate for the elements of $\mathcal{M}_{1}$.

Next, an analogous parallelism (say, $\|^{\prime}$ ) contained in $\mathcal{M}_{1} \times \mathcal{M}_{2}$ can be defined with the property: ' $M_{1} \|^{\prime} M_{2}$ iff $M_{1}^{\infty} \subset M_{2}^{\infty}$ '. Completing the lines in $\mathcal{M}$ by their improper points we obtain the class $\mathcal{T} \cup \mathcal{L}_{1} \cup \mathcal{L}_{2}$ of all the lines of $\mathfrak{P}$ not entirely contained in $\mathcal{H}^{+} \cup \mathcal{H}^{-}$. Finally, it is a trivial trick to re-define the lines on $\mathcal{H}^{+} \cup \mathcal{H}^{-}$as the sets of the improper points of the lines on suitable planes spanned by the lines in $\mathcal{L}_{1}$. Thus, finally, we arrive to the following

Proposition 4.10. The underlying projective space $\mathfrak{P}$ spanned by $\mathcal{H}^{+} \cup \mathcal{H}^{-}$can be defined in terms of the two-holes sliced space $\mathbf{T}\left(\mathcal{H}^{+}, \mathcal{H}^{-}\right)$.

\subsection{Automorphisms}

Analogously as in the affine case, the following is immediate from Proposition 4.10 .

Proposition 4.11. A bijection $F$ of the point set of $\mathbf{T}\left(\mathcal{H}^{+}, \mathcal{H}^{-}\right)$is an automorphism of $\mathbf{T}\left(\mathcal{H}^{+}, \mathcal{H}^{-}\right)$iff $F$ is the restriction of a collineation of the projective space spanned by $\mathcal{H}^{+} \cup \mathcal{H}^{-}$which preserves this spanning set.

After that an analytical characterization of the automorphisms of $\mathbf{M}^{*}(G L(\mathbb{V}))$ follows easily.

Proposition 4.12. The following conditions are equivalent

(i) $F \in \operatorname{Aut}\left(\mathbf{M}^{*}(G L(\mathbb{V}))\right)$;

(ii) there are $\varphi, \psi \in \Gamma L(\mathbb{V})$ and a permutation $\delta$ of $V^{+} \cup V^{-}$such that $\delta(\theta, \theta)=(\theta, \theta), \varphi^{-1} \psi \in G L(\mathbb{V}), F(w)=\delta(w)$ for each $w \in V^{+} \cup V^{-}$, and

(a) $F(u, v)=(\varphi(u), \psi(v))$ for each $u, v \in V \backslash\left(V^{+} \cup V^{-}\right)$, or

(b) $F(u, v)=(\varphi(v), \psi(u))$ for each $u, v \in V \backslash\left(V^{+} \cup V^{-}\right)$.

Note that, since $\mathbf{M}^{*}(G L(\mathbb{V}))$ contains isolated points, $\operatorname{Aut}\left(\mathbf{M}^{*}(G L(\mathbb{V}))\right) \neq$ $\Gamma P(\mathbb{V}) \ltimes G P(\mathbb{V})$ (comp. Proposition 3.11).

Open Access. This article is distributed under the terms of the Creative Commons Attribution License which permits any use, distribution, and reproduction in any medium, provided the original author(s) and the source are credited.

\section{References}

[1] Benz, W.: Vorlesungen über Geometrie der Algebren. Springer, Berlin/Heidelberg/New York (1973)

[2] Gorodowienko, J., Prażmowska, M., Prażmowski, K.: Elementary characterizations of some classes of reducts of affine spaces. J. Geom 89, 17-33 (2008) 
[3] Herzer, A., Meuren, S.: Ein Axiomensystem für partielle affine Räume. J. Geom. 50, 124-142 (1994)

[4] Karzel, H.: Loops related to geometric structures. Quasigroups Related Syst 15, 47-76 (2007)

[5] Karzel, H., Meissner, H.: Geschlitzte Inzidenzgruppen und normale Fastmoduln. Abh. Math. Sem. Univ. Hamburg 31, 69-88 (1967)

[6] Karzel, H., Kosiorek, J., Matraś, A.: Properties of auto- and antiautomorphisms of maximal chain structures and their relations to $i$-perspectivities. Results Math. 50(1-2), 81-92 (2007)

[7] Karzel, H., Kroll, H.-J.: Perspectivities in circle geometries. In: Plaumann, P. et al. (eds.) Geometry - von Staudt's point of view. Proc. NATO Adv. Study Inst. Bad Windsheim 1980, D. Reidel, Dordrecht, pp. 51-99 (1981)

[8] Karzel, H., Pieper, I.: Bericht über geschlitzte Inzidenzgruppen. Jber. Deutsch. Math.-Verein. 70, 70-114 (1970)

[9] Meuren, S.: Partial affine spaces of dimension $\geq 3$. J. Geom. 56, 113-125 (1996)

[10] Wefelscheid, H.: Über die Automorphsmengruppen von Hyperbelstrukturen. Beitr. Geom. Algebra, Proc. Symp. Duisburg, pp. 337-343 (1976), (1977)

Krzysztof Prażmowski and Aneta Sulima

Institute of Mathematics

University of Białystok

ul. Akademicka 2

15-267 Białystok

Poland

e-mail: krzypraz@math.uwb.edu.pl;

anetasulima@wp.pl

Received: January 16, 2011.

Accepted: January 9, 2012. 